\title{
Relaxation in charge-transfer systems with very large tunnel splitting: A semiclassical stochastic approach
}

Cite as: J. Chem. Phys. 113, 11176 (2000); https://doi.org/10.1063/1.1326907

Submitted: 15 August 2000 . Accepted: 27 September 2000 . Published Online: 13 December 2000

J. Casado-Pascual, C. Denk, M. Morillo, and R. I. Cukier

\section{ARTICLES YOU MAY BE INTERESTED IN}

Nonadiabatic semiclassical dynamics in the mixed quantum-classical initial value representation

The Journal of Chemical Physics 148, 102326 (2018); https://doi.org/10.1063/1.5005557

Quantum mechanical transition state theory and a new semiclassical model for reaction rate constants

The Journal of Chemical Physics 61, 1823 (1974); https://doi.org/10.1063/1.1682181

Is the direct observation of electronic coherence in electron transfer reactions possible?

The Journal of Chemical Physics 107, 8397 (1997); https://doi.org/10.1063/1.475040

\section{Lock-in Amplifiers up to $600 \mathrm{MHz}$}
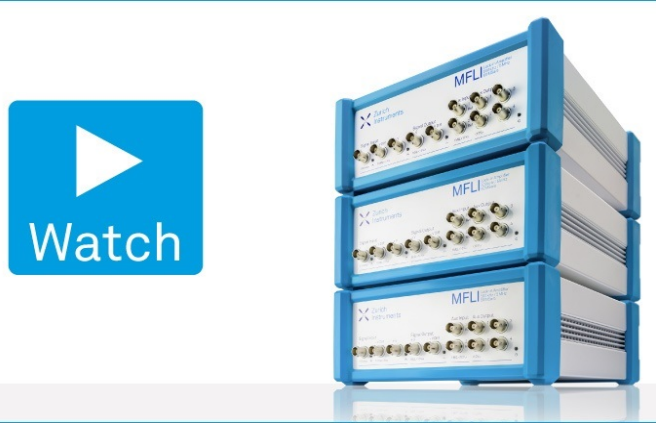

J. Chem. Phys. 113, 11176 (2000); https://doi.org/10.1063/1.1326907

113, 11176

(c) 2000 American Institute of Physics. 


\title{
Relaxation in charge-transfer systems with very large tunnel splitting: A semiclassical stochastic approach
}

\author{
J. Casado-Pascual and C. Denk \\ Física Teórica, Universidad de Sevilla, Apartado de Correos 1065, Sevilla 41080, Spain \\ M. Morillo \\ Física Teórica, Universidad de Sevilla, Apartado de Correos 1065, Sevilla 41080, Spain, \\ and Department of Chemistry and Center for Fundamental Materials Research, \\ Michigan State University, East Lansing, Michigan 48824-1322 \\ R. I. Cukier \\ Department of Chemistry and Center for Fundamental Materials Research, Michigan State University, \\ East Lansing, Michigan 48824-1322
}

(Received 15 August 2000; accepted 27 September 2000)

\begin{abstract}
Electron transfer in strongly coupled systems, appropriate to mixed-valence compounds, is studied to explore the competition between electronic coherence and dissipation. A set of stochastic equations is derived for a spin-boson Hamiltonian with large tunneling coupling matrix element (adiabatic regime) and strong system-bath-coupling. The bath dynamics is treated classically while the quantum character of the system is maintained. The bath dynamics is affected by the system dynamics, the effect being included by a mean-field description, valid for the adiabatic regime. Numerical solutions of the stochastic equations are presented and compared with exact quantum mechanical results. The numerical implementation of the method is straightforward and the long-time behavior of the system can be accessed. Analytic equilibrium solutions for the adiabatic regime are obtained, and we find good agreement between the long-time solution of the stochastic equations and these equilibrium solutions. We examine the dependence of the electronic population on the initial preparation of the bath and find that the proportion between oscillation (coherence) and decay (dissipation) is quite sensitive to this initial condition. (C) 2000 American Institute of Physics. [S0021-9606(00)50448-6]
\end{abstract}

\section{INTRODUCTION}

The phenomena of decoherence and relaxation in dissipative quantum systems are a general problem in condensedphase chemistry and physics. Their study has stimulated the development of several methods of analysis that rely on the regime to be studied. ${ }^{1-18}$ The quantum nature of the interrogated system is always assumed, while the bath that couples to the quantum system may be classical or quantum, depending on the values of its characteristic parameters, the systembath-coupling strength and the temperature, $T$. In many cases, the potential energy relief of the quantum system supports two minima well separated by a barrier. Then, if the temperature is low enough, a reduced description of the quantum system in terms of its first two localized states is adequate. The strength of the coupling between the two states of the system, $\hbar V$, relative to the cutoff energy of the system-bath interaction $\hbar \omega_{c}$ and to $k_{B} T$ are key to the kind of approximation scheme to be used.

In this work, we continue the development of a semiclassical method ${ }^{19-21}$ that we have found to be useful for studying the competition between electronic coherence and dephasing in optical spectroscopy, where a strong external field is applied to a quantum system with just a few degrees of freedom. The quantum system is coupled bi-linearly to a bath of harmonic oscillators, providing a relaxation mechanism. When restricted to a two-state system, the Hamiltonian of the isolated system under the influence of an external field may be written in pseudospin form,

$$
H_{S}=-\frac{\Delta G^{0}}{2} \sigma_{z}+\hbar V(t) \sigma_{x},
$$

where $\Delta G^{0}$ is the free energy difference (asymmetry) and $V(t)$ is proportional to the external field that induces transitions between the two states. In the case of strong field spectroscopy, ${ }^{19,20}$ the parameter $V(t)$ cannot be considered small, and a treatment based on perturbation theory in the strength of the external field is, therefore, not feasible.

When $V(t)$ is large, there are multiple crossings between the states and quantum coherence effects are expected to be important. Then, a semiclassical approach can only be accurate under special circumstances. In a previous study, restricted to a "bath" consisting of one oscillator, we were able to show by numerical calculation and Landau-Zener analysis that semiclassical and quantum mechanical methods will agree in an adiabatic limit that is guaranteed when $V(t)$ is large enough. ${ }^{21}$ The approximation consists of replacing expectation values of the products of system-oscillator operators with products of their expectation values in the exact equations of motion for the spin operators. In this fashion, the differences in the force exerted on the two states is effectively averaged by the fast transitions induced by the large value of $V(t)$. We found that the more classical the oscilla- 


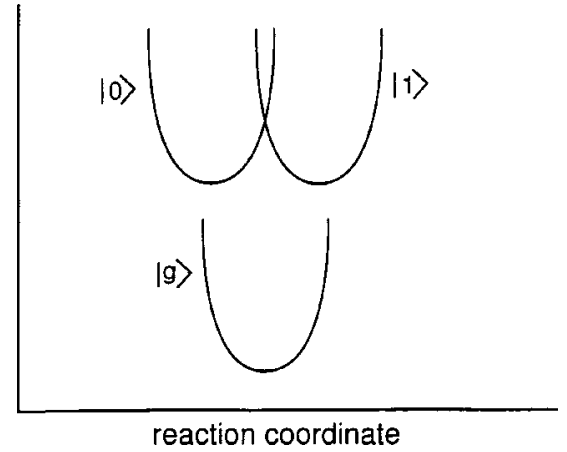

FIG. 1. Schematic solvent free energy surfaces for a photoinitiated electron transfer reaction. The lower surface $|g\rangle$ corresponds to a neutral solute state where the solvent equilibrium is independent of the solute's presence, $\alpha$ $=0$. The solvent equilibrium points for the excited reactant, $|0\rangle$, (product, |1)) states are displaced, relative to this neutral state, as parametrized by the values $\alpha=1(-1)$, cf. Eq. (5). Photochemical excitation $|g\rangle \rightarrow|0\rangle$ initiates the reaction $|0\rangle \leftrightarrow|1\rangle$. If the excited state reaction is fast compared with solvent equilibration times, then an appropriate solvent initial condition is that of the ground equilibrium state, $\alpha=0$.

tor, the more adiabatic the regime. Furthermore, $V(t)$ does not have to be extremely large (in terms of the value of the corresponding Landau-Zener parameter) in order for this semiclassical procedure to work quite well.

A similar system Hamiltonian has been applied by many authors to study electron transfer (ET) reactions, with $V(t)$ $=V$ being the tunneling coupling element. ${ }^{3,2,4,6}$ ET reactions in mixed-valence compounds ${ }^{22,23}$ are characterized by large couplings between the electronic states (large $V$ ) and large system-bath-couplings, rendering perturbation techniques inadequate. The case of large $V$ ET has stimulated several theoretical developments. ${ }^{24-29}$

The spectral density describing the system-bath-coupling is characterized by a cutoff frequency, $\omega_{c}$. For typical polar liquids and experiments at $T=300 \mathrm{~K}, k_{B} T>\hbar \omega_{c} .{ }^{3}$ Thus, a classical treatment of the bath seems adequate for these situations, although the quantum character of the system has to be maintained. Therefore, the one-oscillator semiclassical method just discussed should provide a good starting point for this investigation, as it is best suited to large $V(t)$ and to oscillators that can be treated classically from the thermal $\left(k_{B} T>\hbar \omega_{c}\right)$ and quantum $\left(V(t)>\omega_{c}\right)$ points of view.

Our calculations will be relevant in the analysis of photoinitiated ET reactions ${ }^{30}$ involving three electronic states. Before exciting with a laser pulse, the ground-state solute $(|g\rangle)$ is stable and the solvent is thermally equilibrated to the current electronic charge distribution. A laser pulse puts the solute in an electronically excited state, $|0\rangle$, with the solvent distribution unchanged. This state will be our initial state for a subsequent electron transfer process to a third electronic state, $|1\rangle$. The tunneling matrix element $V$ and the exothermicity $\Delta G^{0}$ characterize the ET between the $|0\rangle$ and $|1\rangle$ states, (cf. Fig. 1). It is important to notice that the solvent is initially in a nonequilibrium situation with respect to the charge distribution of the initial state pertinent to the ET process. There are two possibilities to consider: (a) $V<\omega_{c}$ (fast bath). Here, the bath relaxation toward equilibrium takes place on a time scale that is short compared to the time scale of the electron transfer. Then, the usual expression for the ET rate obtained from a Golden Rule (GR) approach under the assumption of an initially equilibrated solvent [for a large enough reorganization energy $\left(E_{r}\right)$ ] will suffice to describe the process, at least during a relevant time range. On the other hand, (b) $V>\omega_{c}$ (slow bath). The appropriate bath initial condition is the nonequilibrium one. There can be several different stages in the time evolution of the electronic population. For short times, oscillations of the electronic populations, arising from the coupling between the $|0\rangle$ and 1) states can exist. These oscillations are probably too fast to be observed experimentally. ${ }^{30}$ The amplitude of the oscillations will decay due to the solvent fluctuations. As we shall see, the proportion between oscillation and decay in the overall time behavior will depend quite sensitively on the initial condition of the bath. The influence of the initial condition on population evolution has been pointed out by other investigators. 31,24

When $V \ll \omega_{c}$ (the fast bath limit), approximate perturbative schemes have been developed to deal with the dynamics of the reduced density operator for the system. $7,8,15,9,10,32$ Even though $V$ is small, if the system-bath-coupling strength is not too strong and for low enough temperature, coherent oscillations can be found. If $E_{r}$ gets large enough, then a rate regime is obtained where the population decays exponentially with rate constants given by the Marcus-Levich electron transfer (Golden Rule) expression.

Various numerical methods have also been developed to obtain the dynamics of the matrix elements of the reduced system density operator. The Tensor multiplication (TensMult) method of Makri and Makarov ${ }^{33,34}$ is based on a real time path-integral formulation. The practical applicability of the TensMult scheme relies on the fast decay of the memory kernel of the influence functional and this is favored by fast baths (large $\omega_{c}$ ). The TensMult method describes correctly the long-time behavior of dissipative quantum systems; the density matrix has proven to reproduce the correct equilibrium values. In this paper, we mainly focus on slow baths, $V>\omega_{c}$. In this case, it is difficult to obtain reliable results from the TensMult scheme.

Stock $^{35,36}$ recently presented a semiclassical method appropriate to the slow bath limit. In his method, the overall density operator is assumed to factorize at all times into a product of system and bath reduced density operators. The system is described by a finite number of oscillators whose frequencies are distributed in the same form for both diabatic states. Consequently, the scheme is valid up to times $t$ $\ll 1 / \Delta \omega$, where $\Delta \omega$ is the frequency separation between the bath oscillators. In order to access the long-time behavior, a large number of bath oscillators has to be considered, which increases the computational effort. Pechukas et al. ${ }^{18}$ have combined the Makri-Makarov and Stock procedures to give promising results, when the bath spectral density can be partitioned into separate contributions from slow and fast bath modes. They have also implemented a memory equation algorithm for the fast bath part that introduces little loss of accuracy and is numerically efficient. ${ }^{37}$

In this paper we focus on the regime of classical bath dynamics $\left(k_{B} T>\hbar \omega_{c}\right)$ and mainly on the slow baths, $V$ 
$>\omega_{c}$. This second condition precludes the use of perturbation theory in the strength of $V$. Furthermore, we will assume that the system-bath-coupling energy, as characterized by $E_{r}$, is not necessarily small either. Our aim is to construct an approximate stochastic description based on the idea that due to the large value of $V$, transitions among the diabatic states are so fast on the time scale of the bath dynamics, that the bath itself can only follow the system in an average sense, as discussed above for the one-oscillator "bath.",21 This procedure provides a feedback of the electronic population into the dynamics of the bath that is absolutely essential for obtaining reasonable behavior for all times. In particular, the long-time average behavior of the system density matrix agrees with the predictions of an analytic expression for the equilibrium density matrix that we derive in this work. This latter expression is based on tracing out the bath degrees of freedom from the overall canonical density operator in the hightemperature limit. The use of a stochastic description permits the introduction of an infinite number of oscillators in a compact fashion and, as we shall see, leads to a very efficient numerical scheme.

The plan of the rest of the paper is as follows. In Sec. II, we use the spin-boson Hamiltonian as a starting point to derive a set of stochastic equations of motion for the matrix elements of the system density operator and the stochastic process representing the bath reaction coordinate. In Sec. III, we obtain an expression for the equilibrium density matrix of the system that is a generalization to larger values of $V$ of the equilibrium density matrix adequate for small $V$ that is given in terms of the standard reaction free energy $\Delta G^{0}$ and temperature $T{ }^{4}$ In Sec. IV, we discuss the solution of the stochastic equations for a wide range of parameter values. Our concluding remarks are presented in Sec. V.

\section{THE HAMILTONIAN AND EFFECTIVE EQUATIONS OF MOTION}

A model Hamiltonian frequently used to characterize an electron transfer between donor and acceptor centers in condensed media is $1,2,4,3$

$$
\begin{aligned}
\mathcal{H}_{T}= & \sum_{j}\left[\frac{p_{j}^{2}}{2 m_{j}}+\frac{1}{2} m_{j} \omega_{j}^{2}\left(q_{j}-\frac{\gamma_{j}}{m_{j} \omega_{j}^{2}} \sigma_{z}\right)^{2}\right] \\
& -\frac{\Delta G^{0}}{2} \sigma_{z}+\hbar V \sigma_{x} .
\end{aligned}
$$

The two electronic states are denoted by the kets $|0\rangle$ and $|1\rangle$. The solvent is represented by a set of independent harmonic oscillators with shifted centers of oscillations. These shifts depend substantially upon the electronic state of the solute and reflect the differing interaction energies between the two charge distributions of the solute with the solvent. The $m_{j}$ 's and $\omega_{j}$ 's are, respectively, the oscillator masses and frequencies, and the $\gamma_{j}$ 's are the solute-solvent coupling constants. The $\sigma_{i}(i=x, y, z)$ are the Pauli spin operators. Once the solute is reduced to only two active states, it can be described by a spin variable, and we shall use solute and spin language interchangeably. The quantity $\Delta G^{0}$ is the standard free-energy difference between reactants and products. The electronic coupling element $V$ is responsible for the ET between reactants and products.

The equation of motion for the density operator of the total system $\rho_{T}(t)$ is

$$
i \hbar \dot{\rho}_{T}(t)=\left[\mathcal{H}_{T}, \rho_{T}(t)\right] .
$$

We assume that the initial density operator can be represented as

$$
\rho_{T}(0)=\rho_{S}(0) \otimes \rho_{B}(0),
$$

where $\rho_{S}(0)$ describes the initial state of the system, and $\rho_{B}(0)$, the initial density operator for the bath, is of the form

$$
\begin{aligned}
\rho_{B}(0)= & \frac{1}{Z_{B}(\beta)} \exp \left[-\beta \sum_{j}\left(\frac{p_{j}^{2}}{2 m_{j}}+\frac{1}{2} m_{j} \omega_{j}^{2}\right.\right. \\
& \left.\left.\times\left(q_{j}-\frac{\alpha \gamma_{j}}{m_{j} \omega_{j}^{2}}\right)^{2}\right)\right] .
\end{aligned}
$$

Here, $Z_{B}(\beta)$ is the normalization function. This distribution describes an equilibrium ensemble of independent harmonic oscillators with center of oscillations shifted by a quantity parameterized by $\alpha$. This shift reflects the fact that the solvent equilibrium depends upon the solute charge distribution. Adjusting $\alpha$ will permit us to fix the initial bath preparation. The value $\alpha=1$ corresponds to the standard initial condition of nonadiabatic ET reactions, where the bath is equilibrated to the charge distribution of the reactant state, while the value $\alpha=0$ would describe a bath at equilibrium with a neutral reactant state. Finally, we shall consider that the system is initially in a pure state of the form $|\phi\rangle\langle\phi|$.

We will be interested in situations where the temperature is large, compared to the characteristic energy of the bath. Under these conditions, a classical description for the bath dynamics, where the $q_{j}(t)$ are treated as ordinary functions of time, is expected to be a good approximation. On the other hand, the system evolution must be described quantum mechanically. When employing this approximation, a difficulty arises: The coupling term $-\sigma_{z} \Sigma_{j} \gamma_{j} q_{j}$ in the Hamiltonian, Eq. (2), is an operator in the system Hilbert space that influences the bath dynamics. We will introduce an approximation for this term, in order to ensure that the bath variables will maintain their classical character.

Following these considerations, our first step is to introduce an effective Hamiltonian for the bath dynamics of the form

$$
\mathcal{H}_{B}^{\text {eff }}(\mathbf{q}, \mathbf{p}, t)=\sum_{j}\left[\frac{p_{j}^{2}}{2 m_{j}}+\frac{1}{2} m_{j} \omega_{j}^{2} q_{j}^{2}\right]-f(t) \sum_{j} \gamma_{j} q_{j} .
$$

The idea is to approximately incorporate the feedback of the spin dynamics into the bath evolution equations with the term $-f(t) \Sigma_{j} \gamma_{j} q_{j}$, where $f(t)$ is a function of time that contains information about $\sigma_{z}(t)$. The explicit form of $f(t)$ will be specified below. Similar approximations have been considered by other authors to analyze the influence of classical variables on the tunneling of quantum objects. ${ }^{16,35}$ This approximation amounts to neglecting quantum correlations 
among the spin operators in the Heisenberg picture at different times. According to Eq. (6), the Hamiltonian equations for the classical variables $\mathbf{q}(t), \mathbf{p}(t)$ are

$$
\begin{aligned}
& \dot{q}_{j}(t)=\frac{\partial \mathcal{H}_{B}^{\mathrm{eff}}(\mathbf{q}, \mathbf{p}, t)}{\partial p_{j}}=\frac{p_{j}(t)}{m_{j}}, \\
& \dot{p}_{j}(t)=-\frac{\partial \mathcal{H}_{B}^{\mathrm{eff}}(\mathbf{q}, \mathbf{p}, t)}{\partial q_{j}}=-m_{j} \omega_{j}^{2} q_{j}(t)+\gamma_{j} f(t) .
\end{aligned}
$$

This system of differential equations can be easily solved, and the result for $q_{j}(t)$ is

$$
\begin{aligned}
q_{j}(t)= & q_{j}(0) \cos \left(\omega_{j} t\right)+\frac{p_{j}(0)}{m_{j} \omega_{j}} \sin \left(\omega_{j} t\right) \\
& +\frac{\gamma_{j}}{m_{j} \omega_{j}} \int_{0}^{t} d s f(s) \sin \left(\omega_{j}(t-s)\right) .
\end{aligned}
$$

The influence of the dynamical evolution of the bath into the dynamics of the spin subsystem can then be described in terms of an effective Hamiltonian for the spin variables, given by

$$
\begin{aligned}
\mathcal{H}_{S}^{\mathrm{eff}}(t)= & -\left(\frac{\Delta G^{0}}{2}+\sum_{j} \gamma_{j} q_{j}(t)\right) \sigma_{z}+\hbar V \sigma_{x} \\
= & -\frac{\hbar}{2}\left(\frac{\Delta G^{0}}{\hbar}+\eta_{\alpha}(t)+\frac{2 \alpha}{\hbar} \sum_{j} \frac{\gamma_{j}^{2}}{m_{j} \omega_{j}^{2}}\right. \\
& \left.\times \cos \left(\omega_{j} t\right)+\psi(t)\right) \sigma_{z}+\hbar V \sigma_{x},
\end{aligned}
$$

where

$$
\begin{aligned}
\eta_{\alpha}(t)= & \frac{2}{\hbar} \sum_{j} \gamma_{j}\left[\left(q_{j}(0)-\frac{\alpha \gamma_{j}}{m_{j} \omega_{j}^{2}}\right) \cos \left(\omega_{j} t\right)\right. \\
& \left.+\frac{p_{j}(0)}{m_{j} \omega_{j}} \sin \left(\omega_{j} t\right)\right],
\end{aligned}
$$

and

$$
\psi(t)=\frac{2}{\hbar} \int_{0}^{t} d s \sum_{j} \frac{\gamma_{j}^{2}}{m_{j} \omega_{j}} \sin \left(\omega_{j}(t-s)\right) f(s) .
$$

If we introduce the spectral density $J(\omega)$

$$
J(\omega)=\frac{\pi}{2} \sum_{j} \frac{\gamma_{j}^{2}}{m_{j} \omega_{j}} \delta\left(\omega-\omega_{j}\right),
$$

we will have

$$
\sum_{j} \frac{\gamma_{j}^{2}}{m_{j} \omega_{j}^{2}} \cos \left(\omega_{j} t\right)=\frac{2}{\pi} \int_{-\infty}^{\infty} d \omega \frac{J(\omega)}{\omega} \cos (\omega t) .
$$

From now on, we shall assume that the coupling constants and the solvent frequencies are distributed with the Debye spectral density ${ }^{38}$

$$
J(\omega)=\frac{E_{r}}{2} \frac{\omega / \omega_{c}}{1+\left(\omega / \omega_{c}\right)^{2}} \theta(\omega),
$$

and hence

$$
\sum_{j} \frac{\gamma_{j}^{2}}{m_{j} \omega_{j}^{2}} \cos \left(\omega_{j} t\right)=\frac{E_{r}}{2} e^{-\omega_{c} t} .
$$

With this assumption, one obtains

$$
\begin{aligned}
\mathcal{H}_{S}^{\text {eff }}(t)= & -\frac{\hbar}{2}\left(\frac{\Delta G^{0}}{\hbar}+\eta_{\alpha}(t)+\frac{\alpha E_{r}}{\hbar} e^{-\omega_{c} t}+\psi(t)\right) \sigma_{z} \\
& +\hbar V \sigma_{x},
\end{aligned}
$$

and

$$
\psi(t)=\frac{E_{r} \omega_{c}}{\hbar} \int_{0}^{t} d s e^{-\omega_{c}(t-s)} f(s),
$$

which is the solution of the differential equation

$$
\dot{\psi}(t)=-\omega_{c} \psi(t)+\frac{E_{r} \omega_{c}}{\hbar} f(t),
$$

with the initial condition $\psi(0)=0$.

The system density operator $\rho_{S}(t)$ develops in the Schrödinger picture according to the effective dynamical equation

$$
i \hbar \dot{\rho}_{S}(t)=\left[\mathcal{H}_{S}^{\mathrm{eff}}(t), \rho_{S}(t)\right],
$$

with the initial condition $|\phi\rangle\langle\phi|$. This differential equation depends on the initial values $\{\mathbf{q}(0), \mathbf{p}(0)\}$ through the stochastic process $\eta_{\alpha}(t)$. According to Eqs. (5) and (11), $\eta_{\alpha}(t)$ is the stationary, Markovian and Gaussian process known as the Ornstein-Uhlenbeck (OU) process, ${ }^{39}$ and hence it is fully specified by its average

$$
\left\langle\eta_{\alpha}(t)\right\rangle_{B}=0
$$

and its second moment

$$
\left\langle\eta_{\alpha}(t) \eta_{\alpha}\left(t^{\prime}\right)\right\rangle_{B}=\Delta^{2} e^{-\omega_{c}\left|t-t^{\prime}\right|},
$$

where \langle\rangle$_{B}$ means the average over the bath degrees of freedom, and $\Delta=\sqrt{2 E_{r} /\left(\hbar^{2} \beta\right)}$. This process can be generated by the solution of the stochastic differential equation

$$
\dot{\eta}_{\alpha}(t)=-\omega_{c} \eta_{\alpha}(t)+w(t),
$$

where $w(t)$ is a zero-average white noise with correlation function $\langle w(t) w(s)\rangle=2 \Delta^{2} \omega_{c} \delta(t-s)$. The initial value $\eta_{\alpha}$ $=\eta_{\alpha}(0)$ is distributed according to the Gaussian distribution

$$
P\left(\eta_{\alpha}\right)=\frac{1}{\sqrt{2 \pi \Delta^{2}}} e^{-\left(\eta_{\alpha}^{2} / 2 \Delta^{2}\right)} .
$$

It is important to note that, for a given realization of the OU process $\eta_{\alpha}(t)$, the solution of Eq. (20) can be formally written as

$$
\rho_{S}(t)=U(t)|\phi\rangle\langle\phi| U^{\dagger}(t),
$$

where $U(t)$ is the unitary evolution operator that satisfies the equation

$$
i \hbar \dot{U}(t)=\mathcal{H}_{S}^{\text {eff }}(t) U(t) .
$$

The initial condition is $U(0)=\mathcal{I}$, with $\mathcal{I}$ the identity operator in the system Hilbert space. Hence, Eq. (20) maintains the normalization condition $\operatorname{Tr}_{S}\left(\rho_{S}(t)\right)=1$, where $\operatorname{Tr}_{S}$ is the 
trace in the system Hilbert space. As $\rho_{S}(t)$ is an operator in the system Hilbert space, it can be expanded in terms of the identity and Pauli operators

$$
\rho_{S}(t)=\frac{1}{2}\left[\mathcal{I}+x(t) \sigma_{x}+y(t) \sigma_{y}+z(t) \sigma_{z}\right] .
$$

The above system of equations can be closed with a choice for the function $f(t)$. Motivated by the large $V$ adiabatic regime, where the coupling is large, compared with the bath fluctuation speed, we choose ${ }^{21}$

$$
f(t)=\operatorname{Tr}_{S}\left(\rho_{S}(t) \sigma_{z}\right)=\left\langle 0\left|\rho_{S}(t)\right| 0\right\rangle-\left\langle 1\left|\rho_{S}(t)\right| 1\right\rangle=z(t) .
$$

Using Eqs. (27) and (28), in conjunction with Eqs. (20) and (19), we get

$$
\begin{aligned}
\dot{x}(t)= & {\left[\frac{\Delta G^{0}}{\hbar}+\eta_{\alpha}(t)+\frac{\alpha E_{r}}{\hbar} e^{-\omega_{c} t}+\psi(t)\right] y(t), } \\
\dot{y}(t)= & -\left[\frac{\Delta G^{0}}{\hbar}+\eta_{\alpha}(t)+\frac{\alpha E_{r}}{\hbar} e^{-\omega_{c} t}+\psi(t)\right] x(t) \\
& -2 V z(t), \\
\dot{z}(t)= & 2 V y(t), \\
\dot{\psi}(t)= & -\omega_{c} \psi(t)+\frac{E_{r} \omega_{c}}{\hbar} z(t) .
\end{aligned}
$$

These differential equations, together with Eq. (23), constitute the set of stochastic differential equations that yield the evolution of $\rho_{S}(t)$. They must be solved with the initial conditions $\quad x(0)=2 \operatorname{Re}\left[\langle 0 \mid \phi\rangle\langle 1 \mid \phi\rangle^{*}\right], \quad y(0)=-2 \operatorname{Im}[\langle 0 \mid \phi\rangle$ $\left.\times\langle 1 \mid \phi\rangle^{*}\right], z(0)=2|\langle 0 \mid \phi\rangle|^{2}-1, \psi(0)=0$, and Eq. (24).

Obviously, the actual system density operator is the one that is obtained after averaging over the bath variables, $\left\langle\rho_{S}(t)\right\rangle$, or, equivalently, after averaging over the realizations of the OU process $\eta_{\alpha}(t)$. If the initial system density operator does not correspond to a pure state but to a mixture of pure states of the form $\rho_{S}(0)=c_{1}\left|\phi_{1}\right\rangle\left\langle\phi_{1}\left|+c_{2}\right| \phi_{2}\right\rangle$ $\times\left\langle\phi_{2}\right|$, with $c_{1}+c_{2}=1$, the method we have just described must be applied to each pure state independently, obtaining $\left\langle\rho_{S}^{(j)}(t)\right\rangle(j=1,2)$, and the final result will be

$$
\left\langle\rho_{S}(t)\right\rangle=c_{1}\left\langle\rho_{S}^{(1)}(t)\right\rangle+c_{2}\left\langle\rho_{S}^{(2)}(t)\right\rangle .
$$

Finally, for the purposes of numerical analysis and presentation of the results, it is convenient to define the dimensionless parameters $\tilde{t}=\omega_{c} t, \quad \widetilde{E}_{r}=E_{r} /\left(\hbar \omega_{c}\right), \quad \widetilde{T}$ $=\left(\beta \hbar \omega_{c}\right)^{-1}, \Delta \widetilde{G}^{0}=\Delta G^{0} /\left(\hbar \omega_{c}\right), \widetilde{V}=V / \omega_{c}$, and the dimensionless functions $\tilde{\eta}_{\alpha}(\tilde{t})=\eta_{\alpha}\left(\tilde{t} / \omega_{c}\right) / \Delta$ and $\widetilde{\psi}(\tilde{t})$ $=\hbar \psi\left(\tilde{t} / \omega_{c}\right) / E_{r}$. Using these definitions, Eqs. (29)-(32) and Eq. (23) can be expressed as

$$
\begin{aligned}
\dot{x}(\tilde{t})= & {\left[\Delta \widetilde{G}^{0}+\left(2 \widetilde{E}_{r} \widetilde{T}\right)^{1 / 2} \widetilde{\eta}_{\alpha}(\widetilde{t})+\alpha \widetilde{E}_{r} e^{-\tilde{t}}\right.} \\
& \left.+\widetilde{E}_{r} \widetilde{\psi}(\tilde{t})\right] y(\tilde{t}), \\
\dot{y}(\widetilde{t})= & -\left[\Delta \widetilde{G}^{0}+\left(2 \widetilde{E}_{r} \widetilde{T}\right)^{1 / 2} \widetilde{\eta}_{\alpha}(\widetilde{t})+\alpha \widetilde{E}_{r} e^{-\tilde{t}}\right. \\
& \left.+\widetilde{E}_{r} \widetilde{\psi}(\tilde{t})\right] x(\widetilde{t})-2 \widetilde{V} z(\tilde{t}), \\
\dot{z}(\tilde{t})= & 2 \widetilde{V}_{y}(\widetilde{t}),
\end{aligned}
$$

$$
\begin{aligned}
& \dot{\tilde{\psi}}(\tilde{t})=-\widetilde{\psi}(\tilde{t})+z(\widetilde{t}), \\
& \dot{\tilde{\eta}}_{\alpha}(\tilde{t})=-\tilde{\eta}_{\alpha}(\tilde{t})+\tilde{w}(\widetilde{t}),
\end{aligned}
$$

where $\tilde{w}(\tilde{t})$ is a zero-average white noise with correlation function $\left\langle\tilde{w}(\tilde{t}) \tilde{w}\left(\tilde{t}^{\prime}\right)\right\rangle=2 \delta\left(\tilde{t}-\tilde{t}^{\prime}\right)$, and the initial value $\tilde{\eta}_{\alpha}(0)$ is distributed according to Eq. (24) with $\Delta=1$. The interpretation of the above equations of motion is as follows: Eq. (38) describes the autonomous part of the bath dynamics that gives rise to a level broadening in the effective Hamiltonian Eq. (17). The initial displacement provides the exponentially decaying terms proportional to $\alpha E_{r}$ in the effective Hamiltonian and the corresponding equations of motion. The dynamics of the isolated system is determined by the parameters $\widetilde{V}$ and $\Delta \widetilde{G}^{0}$. Equation (37) describes the part of the bath dynamics affected by the system dynamics in terms of the population difference $z(\tilde{t})$ and enters the system equations as a feedback term.

The presence of the feedback $\widetilde{\psi}(\widetilde{t})$ in the equations of motion is essential for the system to reach an equilibrium that depends on $\Delta \widetilde{G}^{0}$. When the feedback is ignored $[\widetilde{\psi}(\tilde{t})$ $=0]$, we find, by numerically solving Eqs. (34)-(38), that $\langle z(\tilde{t})\rangle \rightarrow 0$ for all values of $\Delta \widetilde{G}^{0}$. The inclusion of $\widetilde{\psi}(\tilde{t})$ allows the population difference to relax to a value that depends on $\Delta \widetilde{G}^{0}$, only for $\Delta \widetilde{G}^{0}=0$ do we obtain $\lim _{\tilde{t} \rightarrow \infty}\langle z(\tilde{t})\rangle \rightarrow 0$. An analytic analysis of the behavior of the equations of motion seems difficult, as the noise term $\tilde{\eta}_{\alpha}(\tilde{t})$ is multiplicative. We have not succeeded in finding closed equations for the mean values.

\section{THE EQUILIBRIUM DENSITY OPERATOR}

In this section we shall obtain an approximate expression for the systems's equilibrium density operator that should be correct for a classical bath. Let us stress that, in general, especially when the bath is not classical, there is no rigorous way to disentangle the system's density operator from that of the bath. Let us assume that the total equilibrium density operator has the canonical form

$$
\rho_{T}^{\mathrm{eq}}(\beta)=\frac{1}{Z_{T}(\beta)} e^{-\beta \mathcal{H}_{T}},
$$

where $\mathcal{H}_{T}$ is the total Hamiltonian given by Eq. (2) and $Z_{T}(\beta)$ is the total partition function. Our goal now is to trace out the bath variables by considering them as classical quantities. Hence, in the definition of the reduced density operator of the system at equilibrium, we must replace the trace in the bath Hilbert space, $\operatorname{Tr}_{B}$, by an integral over phase space

$$
\rho_{S}^{\mathrm{eq}}(\beta)=\frac{1}{Z(\beta)} \operatorname{Tr}_{B}\left[e^{-\beta \mathcal{H}_{T}}\right] \rightarrow \frac{1}{Z(\beta)} \iint d \mathbf{q} d \mathbf{p} e^{-\beta \mathcal{H}_{T}} .
$$

Integrating over the $\mathbf{p}$ variables, one finds

$$
\begin{aligned}
\rho_{S}^{\mathrm{eq}}(\beta)= & C(\beta) \iint d \mathbf{q} e^{-\beta / 2 \Sigma_{j} m_{j} \omega_{j}^{2} q_{j}^{2}} \\
& \times e^{(\beta / 2)\left(\left(\Delta G^{0}+2 \Sigma_{j} \gamma_{j} q_{j}\right) \sigma_{z}-2 \hbar V \sigma_{x}\right)},
\end{aligned}
$$


where $C(\beta)$ is a normalization factor.

If we define $\eta=(2 / \hbar) \Sigma_{j} \gamma_{j} q_{j}$, then the integration over the $\mathbf{q}$ variables can be easily carried out by inserting an appropriate delta function to obtain

$$
\begin{aligned}
\rho_{S}^{\mathrm{eq}}(\beta)= & C(\beta) \iint d \mathbf{q} e^{-(\beta / 2) \Sigma_{j} m_{j} \omega_{j}^{2} q_{j}^{2}} \int_{-\infty}^{\infty} d \eta \\
& \times \delta\left(\eta-(2 / \hbar) \Sigma_{j} \gamma_{j} q_{j}\right) e^{(\beta / 2)\left(A(\eta) \sigma_{z}-2 \hbar V \sigma_{x}\right)} \\
= & C^{\prime}(\beta) \int_{-\infty}^{\infty} d \eta P(\eta) e^{(\beta / 2)\left(A(\eta) \sigma_{z}-2 \hbar V \sigma_{x}\right)},
\end{aligned}
$$

where $C^{\prime}(\beta)$ is another normalization constant, $A(\eta)$ $=\Delta G^{0}+\hbar \eta$, and $P(\eta)$ is given in Eq. (24).

Using the identity ${ }^{40}$

$$
\begin{aligned}
e^{(\beta / 2)\left(A(\eta) \sigma_{z}-2 \hbar V \sigma_{x}\right)}= & \cosh (\beta E(\eta)) \mathcal{I} \\
& +\frac{\sinh (\beta E(\eta))}{E(\eta)}\left[\frac{A(\eta)}{2} \sigma_{z}-\hbar V \sigma_{x}\right],
\end{aligned}
$$

where $E(\eta)=\left[(A(\eta) / 2)^{2}+(V \hbar)^{2}\right]^{1 / 2}$, the normalization constant $C^{\prime}(\beta)$ can be expressed in the form

$$
C^{\prime}(\beta)=\left(2 \int_{-\infty}^{\infty} d \eta P(\eta) \cosh (\beta E(\eta))\right)^{-1} .
$$

Hence, if we write $\rho_{S}^{\mathrm{eq}}(\beta)=\frac{1}{2}\left[\mathcal{I}+X^{\mathrm{eq}}(\beta) \sigma_{x}+Y^{\mathrm{eq}}(\beta) \sigma_{y}\right.$ $\left.+Z^{\mathrm{eq}}(\beta) \sigma_{z}\right]$, we obtain

$$
\begin{aligned}
& X^{\mathrm{eq}}(\beta)=-\hbar V \frac{\int_{-\infty}^{\infty} d \eta P(\eta) \sinh (\beta E(\eta)) / E(\eta)}{\int_{-\infty}^{\infty} d \eta P(\eta) \cosh (\beta E(\eta))}, \\
& Y^{\mathrm{eq}}(\beta)=0, \\
& Z^{\mathrm{eq}}(\beta)=\frac{\int_{-\infty}^{\infty} d \eta P(\eta) A(\eta) \sinh (\beta E(\eta)) / E(\eta)}{2 \int_{-\infty}^{\infty} d \eta P(\eta) \cosh (\beta E(\eta))},
\end{aligned}
$$

or, in dimensionless form,

$$
\begin{aligned}
& X^{\mathrm{eq}}(\widetilde{T})=-\widetilde{V} \frac{\int_{-\infty}^{\infty} d \tilde{\eta} P(\tilde{\eta}) \sinh (\tilde{E}(\tilde{\eta}) / \widetilde{T}) / \widetilde{E}(\tilde{\eta})}{\int_{-\infty}^{\infty} d \tilde{\eta} P(\tilde{\eta}) \cosh (\widetilde{E}(\tilde{\eta}) / \widetilde{T})}, \\
& Y^{\mathrm{eq}}(\widetilde{T})=0, \\
& Z^{\mathrm{eq}}(\widetilde{T})=\frac{\int_{-\infty}^{\infty} d \tilde{\eta} P(\tilde{\eta}) \tilde{A}(\tilde{\eta}) \sinh (\widetilde{E}(\tilde{\eta}) / \widetilde{T}) / \widetilde{E}(\tilde{\eta})}{2 \int_{-\infty}^{\infty} d \tilde{\eta} P(\tilde{\eta}) \cosh (\tilde{E}(\tilde{\eta}) / \widetilde{T})},
\end{aligned}
$$

where $\quad \tilde{\eta}=\eta / \Delta, \quad \tilde{A}(\tilde{\eta})=\Delta \widetilde{G}^{0}+\left(2 \widetilde{E}_{r} \widetilde{T}\right)^{1 / 2} \tilde{\eta}, \quad \tilde{E}(\tilde{\eta})$ $=\left[(\tilde{A}(\tilde{\eta}) / 2)^{2}+(\tilde{V})^{2}\right]^{1 / 2}$, and $P(\tilde{\eta})$ is the Gaussian distribution defined in Eq. (24) with $\Delta=1$. These expressions can be evaluated numerically quite readily. In the following section, we will compare the equilibrium results with those obtained from the long-time solution of the dynamical equations.

\section{RESULTS AND DISCUSSION}

In this section, we will present numerical results based on our equations of motion that illustrate the competition between electronic coherence and relaxation induced by the system-bath-coupling. Comparisons with other available
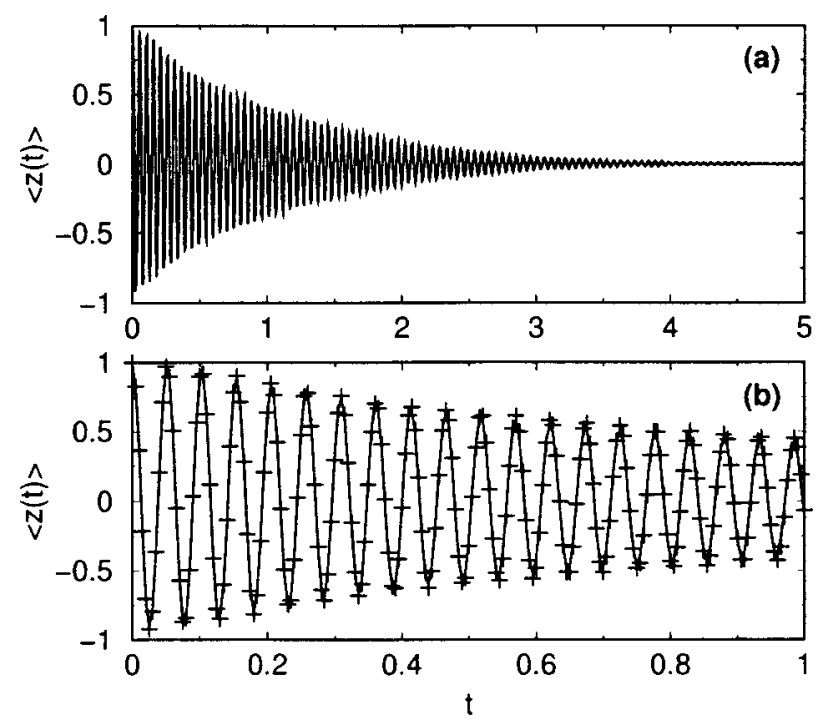

FIG. 2. (a) $\langle z(t)\rangle$ for $\widetilde{V}=60, \widetilde{E}_{r}=80.0, \widetilde{T}=4.0, \Delta \widetilde{G}^{0}=0.0$ and $\alpha=0.0$. (All quantities are given in dimensionless units relative to the bath characteristic frequency, $\omega_{c}$.) For this large value of $\widetilde{V}$, there are fast oscillations whose amplitude slowly decays to zero, the equal population state. (b) Comparison of our semiclassical stochastic method (solid line) and the semiclassical method of Stock (plus signs).

methods are made, when feasible. Throughout this section we will use the dimensionless form of the equations, Eqs. (34)-(38), i.e., all frequencies are scaled with respect to the bath frequency $\omega_{c}$. If $\omega_{c}=50.0 \mathrm{~cm}^{-1}$, then a $\widetilde{V}$ in the range $0.01-100.0$ corresponds to $V=0.5-5000.0 \mathrm{~cm}^{-1}$, which covers the range of typical electronic couplings. The values of reorganization energies, $\widetilde{E}_{r}$, used below will be characteristic of nonpolar and polar solvents. For convenience, we will drop the tilde in what follows.

Our set of stochastic equations has been integrated using a stochastic Runge-Kutta algorithm of second order in the noise and the deterministic parts. ${ }^{41}$ For each initial value of $\eta_{\alpha}$, drawn from the Gaussian distribution Eq. (24), the equations are solved for a realization of the white noise $w(t)$. Mean values have been obtained with $N_{\text {tr }}=10000$ trajectories. The integration time step has to be matched to the system parameters, especially for large values of $V$ and $\Delta G^{0}$. The system is initially prepared with $x(0)=y(0)=0$, and $z(0)=1$, corresponding to unit population of state $|0\rangle$ [cf. Fig. (1)].

In panel (a) of Fig. 2 we display the behavior of the population difference $\langle z(t)\rangle$ for $\Delta G^{0}=0.0, V=60.0, E_{r}$ $=80.0, T=4.0$, corresponding to the strictly adiabatic limit $V \gg \omega_{c}=1.0$, and for a nonequilibrium initial condition, $\alpha$ $=0.0$. This figure shows that the decay is slow and highly oscillatory. The decay should be to zero since $\Delta G^{0}=0$ (see below).

The semiclassical method of Stock ${ }^{35}$ is equivalent to our method in the limit of an infinite number of bath oscillators. One would, therefore, expect perfect agreement between the two methods for time intervals $t \ll 1 / \Delta \omega$, where $\Delta \omega$ is the separation in frequency between the finite number of oscillators used in Stock's method. We have integrated Stock's equations with $N=400$ oscillators with frequencies uni- 
formly distributed in the interval $\left[\omega_{\min }, \omega_{\max }\right]=\left[10^{-3}, 10\right]$ for a Debye spectral density. The initial conditions for the bath oscillators are obtained from the classical action-angle variables

$$
\begin{aligned}
& q_{j}(0)=\sqrt{2 n_{j}+1} \sin \left(\phi_{j}\right), \\
& p_{j}(0)=\sqrt{2 n_{j}+1} \cos \left(\phi_{j}\right),
\end{aligned}
$$

with random phases $\phi_{j}$ and quantum numbers $n_{j}$ distributed according to the Boltzmann distribution. For the integration of the system of equations in Stock's model, a fixed time step Runge-Kutta method of second order has been used, integrating $N_{\text {tr }}=2500$ trajectories in order to obtain converged results.

In panel (b) of Fig. 2, we compare our results with those obtained using the scheme of Stock, which is also designed to be accurate in the adiabatic limit. As expected, the agreement between the two methods is very good in the observed time interval. The difference for larger times is due to the limited number of oscillators in the method of Stock; the agreement may be further improved by increasing this number.

The CPU time needed for 1000 trajectories on a Pentium II (400 MHz) was $1 \mathrm{~min}$ for our method and $290 \mathrm{~min}$ for the method of Stock with the abovementioned parameters. This difference stems from the fact that, in Stock's method, the equations of motion for all $N$ bath oscillators have to be integrated. This results in a system of $2 N+4$ ordinary first order differential equations, rendering the numerical solution of this problem time consuming for a large number of bath oscillators. On the other hand, the stochastic scheme requires the numerical solution of a set of only five stochastic first order differential equations. Although the necessary CPU time for Stock's method could be reduced by employing faster integration algorithms such as the velocity Verlet algorithm, ${ }^{42}$ the stochastic method will always be less numerically demanding.

When $E_{r}$ is quite large, damping of $\langle z(t)\rangle$ will be in evidence, as shown in Fig. 3. Even though $V$ is large, the initial decay and oscillation goes over to exponential relaxation. Note that, for the equilibrium initial condition $(\alpha$ $=1.0$ ) used here, the solvent configurations are centered around the reactant well minimum, such that their weight around the crossing point of the electronic surfaces is small, thus de-emphasizing the $V$-dependent oscillatory behavior of $\langle z(t)\rangle$. The ratio of the slopes of the decays for the two values of $V$ is about 1.5 , indicating that they are not independent of the strength of the electronic coupling. That is, if one were using a Marcus adiabatic ET rate expression, ${ }^{5}$ consonant with a large value of $V$, the rate ratio would be one, since the reorganization energy is fixed here. In contrast with the equilibrium initial condition used to construct Fig. 3, Fig. 4 shows that if we use the nonequilibrium initial condition $(\alpha=0.0)$ the oscillations are much more prominent and the decay behavior, while still evident, starts from a much smaller amplitude than for $\alpha=1.0$. The much larger weight of solvent configurations around the crossing point of the surfaces for this initial condition produces this behavior. We also display in Fig. $4\langle z(t)\rangle$ for the larger $E_{r}$ value of 40.0 .

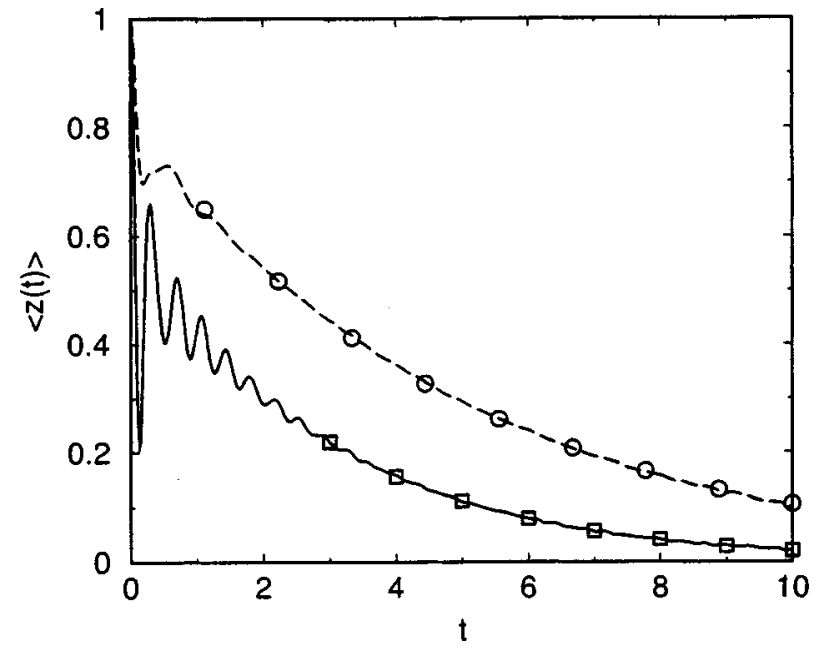

FIG. 3. $\langle z(t)\rangle$ for $\widetilde{V}=4.0, \widetilde{E}_{r}=20.0, \widetilde{T}=2.0, \Delta \widetilde{G}^{0}=0.0$ and $\alpha=1.0$ (dashed line). The initial condition has the bath equilibrated to the reactant state. There is an initial fast decay due to the relatively large value of $\widetilde{V}$ followed by an exponential decay, due to the large reorganization energy, $\widetilde{E}_{r}$. Since initially there is little solvent population at the crossing point of the surfaces (see text), the crossover to relaxation behavior dominates. For a larger electronic coupling, $\widetilde{V}=8.0$ (solid line), there is more initial oscillation, but $\langle z(t)\rangle$ still goes over to an exponential decay, with a decay constant about 1.5 times larger than that of the $\widetilde{V}=4.0$ case. The circles (boxes) are exponential fits to the data for $\widetilde{V}=4.0(\widetilde{V}=8.0)$.

The decay is similar to the $E_{r}=20.0$ case, with a larger initial amplitude reflecting the increased solvent-solute interaction. The feature of more pronounced oscillations for a nonequilibrium initial condition agrees with the findings of other investigators. ${ }^{24,25}$

Now we consider the predictions made by our stochastic equations for the long-time average behavior, by comparing their results with the analytic equilibrium values obtained by

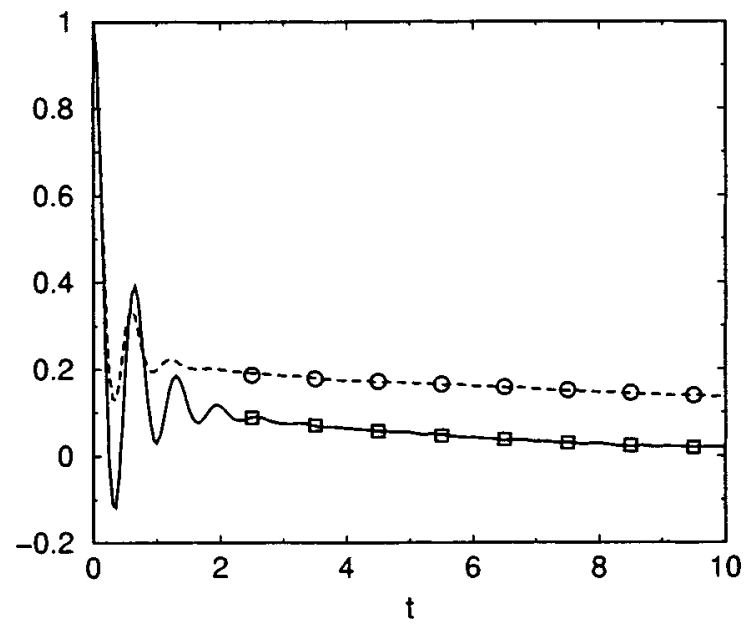

FIG. 4. $\langle z(t)\rangle$ for $\widetilde{V}=4.0, \widetilde{E}_{r}=20.0$ (solid line) and 40.0 (dashed line) with $\widetilde{T}=2.0, \widetilde{G}^{0}=0.0$ and $\alpha=0.0$. For the nonequilibrium initial condition, the initial solvent population at the surface crossing is much larger than for the data in Fig. 3 and the initial decay is more oscillatory. Nevertheless, $\langle z(t)\rangle$ does go over to an exponential decay, with the larger $\widetilde{E}_{r}$ case starting from a larger value. The boxes (circles) are exponential fits to the data for $\widetilde{E}_{r}$ $=20.0\left(\widetilde{E}_{r}=40.0\right)$. 


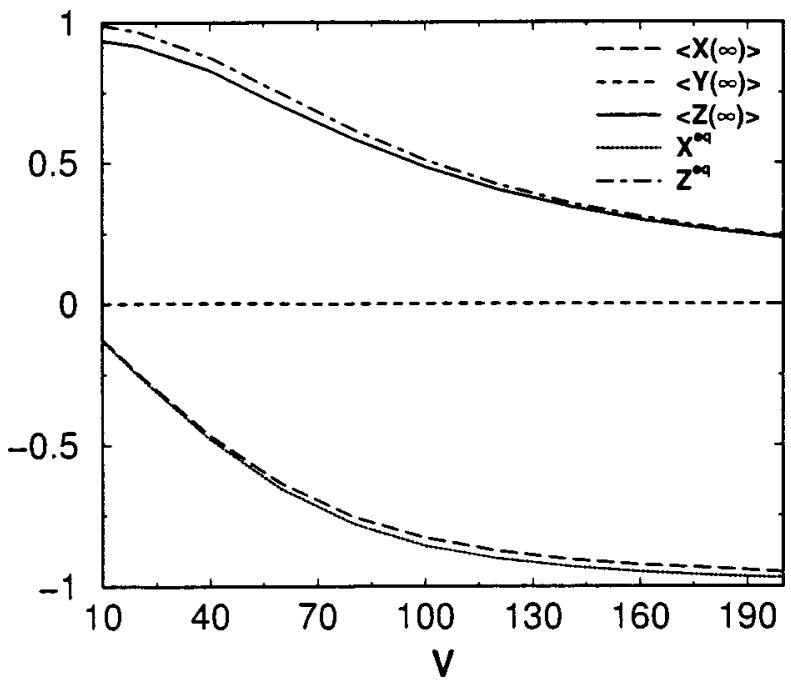

FIG. 5. The dependence of $\langle x(\infty)\rangle,\langle y(\infty)\rangle$, and $\langle z(\infty)\rangle$ on the tunneling coupling element $V$ for fixed $\widetilde{T}=4.0, \widetilde{E}_{r}=80.0$, and $\Delta \widetilde{G}_{0}=80.0$, as obtained from the numerical solution of the stochastic equations, and the dependence of $X^{\mathrm{eq}}, Y^{\mathrm{eq}}, Z^{\mathrm{eq}}$ obtained from the equilibrium distribution. All the quantities are in dimensionless form.

quadrature from Eqs. (48)-(50). Note that the stochastic equation formulation is not computationally demanding, which permits us to readily obtain the long-time behavior. In Fig. 5, we compare the analytic and numerical results for values of $V$ that are sufficiently large to guarantee the accuracy of our theory. In Fig. 6, the comparison is made as a function of $\Delta G^{0}$. Clearly, the stochastic equations provide a good account of the equilibrium behavior that is predicted by Eqs. (48)-(50). However, it must be pointed out that there is no a priori reason that demands coincidence between these results, as we discuss further in Sec. V. The key feature of the stochastic equations responsible for obtaining nonzero $\langle z(\infty)\rangle$ values, as must be the case when $\Delta G^{0}$ is not zero, is the dynamical feedback from the system to the bath, as em-

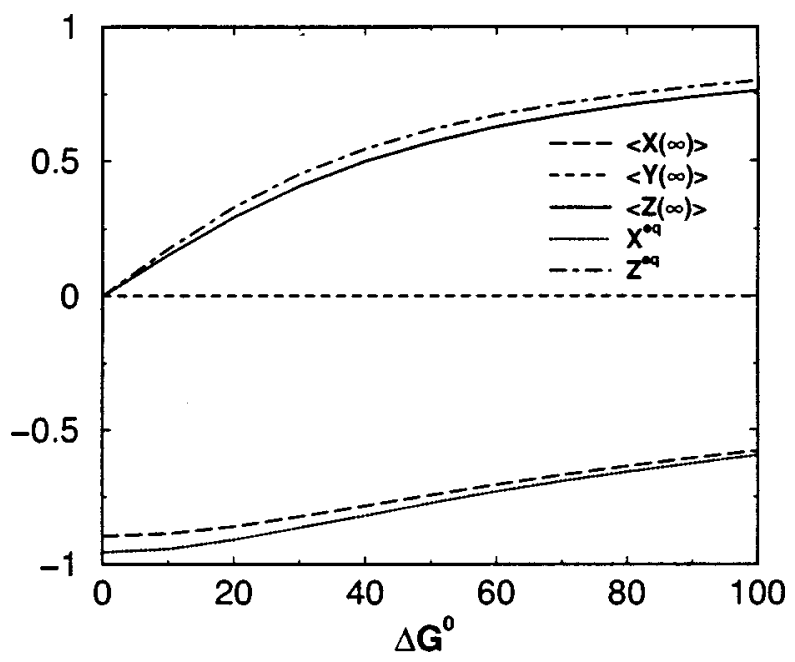

FIG. 6. The dependence of $\langle x(\infty)\rangle,\langle y(\infty)\rangle$ and $\langle z(\infty)\rangle$, on $\Delta \widetilde{G}^{0}$ for fixed $\widetilde{T}=4.0, \widetilde{E}_{r}=80.0$, and $\widetilde{V}=60.0$, as obtained from the numerical solution of the stochastic equations, and the dependence of $X^{\mathrm{eq}}, Y^{\mathrm{eq}}$ and $Z^{\mathrm{eq}}$, obtained from the equilibrium distribution. All the quantities are in dimensionless form.
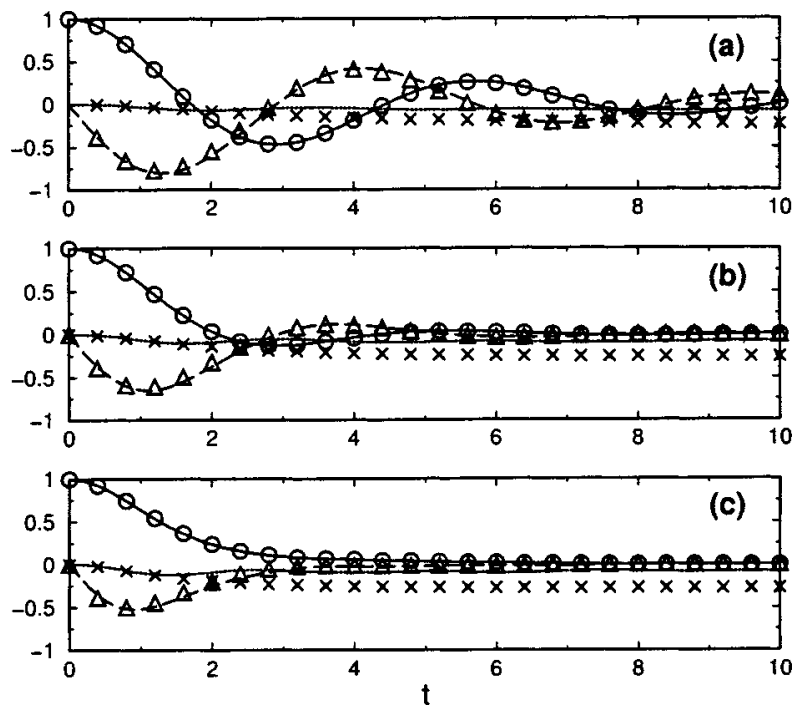

FIG. 7. Comparison of the semiclassical stochastic method (lines) and the numerically exact tensor multiplication scheme of Makri and Makarov (symbols). (a) $\widetilde{V}=0.5, \widetilde{E}_{r}=0.2, \widetilde{T}=2.0, \Delta \widetilde{G}^{0}=0.0$, and $\alpha=1.0$. (b) As in (a) but $E_{r}=0.5$. (c) As in (a) but $\widetilde{E}_{r}=1.0$. The agreement between $\langle z(t)\rangle$ and $\langle y(t)\rangle$ is excellent for all times, while the $\langle x(t)\rangle$ behavior is only good at short times. This deviation reflects the breakdown of our adiabatic approximation as $\widetilde{V}$ gets sufficiently small. Nevertheless, the population behavior is well described by our method. As $\widetilde{E}_{r}$ increases the $\langle z(t)\rangle$ behavior changes from an underdamped to an overdamped oscillatory decay.

bodied in Eqs. (29) -(32), (28), and (18). If inside the integral defining $\psi(t)$ in Eqs. (18) and (28) the value of $z(s)$ were set to one, the resulting equations would lead to $\langle z(\infty)\rangle=0$ for all $\Delta G^{0}$ values. This approximation would amount to neglecting the influence of the dynamics of the solute (spin) on the solvent (bath) fluctuations. Due to this dynamical coupling, the evolution of the bath cannot be specified by an autonomous stochastic process.

Our stochastic equations are appropriate when $V$ is sufficiently large compared with the other characteristic frequencies; nevertheless, it is interesting to investigate if they give reasonable results for smaller values of $V$. To carry out this task, we will compare our results with numerical results obtained using the tensor multiplication scheme of Makri and Makarov. ${ }^{33,34}$ The practical implementation of this scheme requires $V \leqslant \omega_{c}$, in order to obtain convergence when a reasonable number of nonlocal memory terms are taken into account $\left(\Delta k_{\max } \approx 10\right)$. In what follows, we have chosen parameter values where convergence can be achieved with $\Delta k_{\max }=9$ in the TensMult algorithm, so that the results of the TensMult algorithm can be considered exact.

In Fig. 7, we study the behavior of $\langle x\rangle,\langle y\rangle$, and $\langle z\rangle$ for the unbiased case $\Delta G^{0}=0$ with $V=0.25$ and $T=2.0$ for three different values of the reorganization energy $E_{r}$ $=0.2,0.5$, and 1.0. These parameter values correspond to relatively high-temperature and weak system-bath-coupling. For all system-bath-couplings we find very good agreement between the stochastic equation and the TensMult schemes for $\langle z\rangle$. A similar conclusion was reached by Golosov et al. ${ }^{18}$ in comparing the Stock and TensMult schemes. We have also examined $\langle y\rangle$ and find good agreement though the deviations are larger here. However, we see that for $\langle x\rangle$, the 


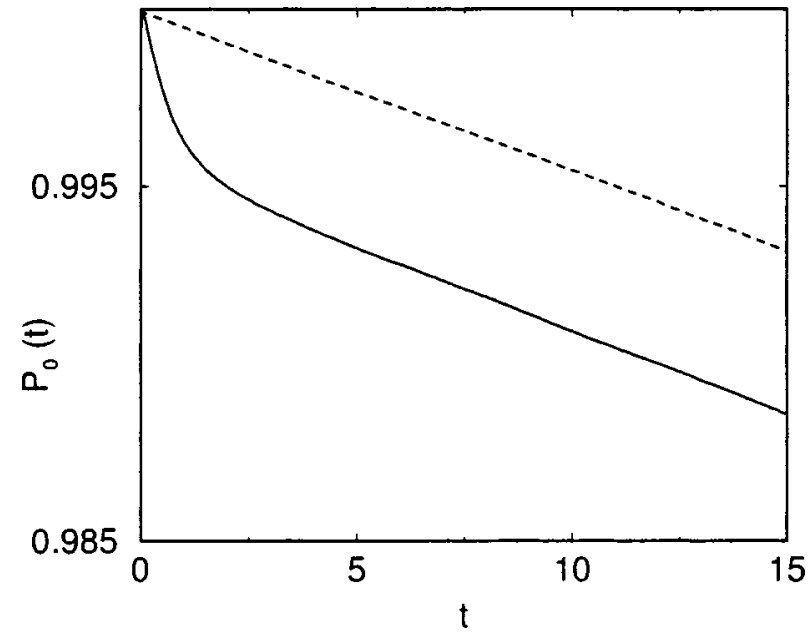

FIG. 8. Short time behavior of the reactant state population, $P_{0}(t)$, for $\widetilde{V}$ $=0.2, \widetilde{E}_{r}=40.0, \widetilde{T}=4.0, \Delta \widetilde{G}^{0}=0.0$, and $\alpha=1.0$ (dashed line) and $\alpha=0.0$ (solid line). The essentially immediate exponential decay for $\alpha=1.0$ and the exponential decay after the transient for $\alpha=0.0$, the nonequilibrium initial condition, over a time interval where the population decay is small, occurs with close to the same decay rate. This decay rate agrees with the Golden Rule value, cf. Eq. (52) in the text, indicating the validity of a rate regime for sufficiently small $\widetilde{V}$ and large $\widetilde{E}_{r}$.

semiclassical method only agrees with the purely quantum mechanical results for short times. The error in the long-time behavior of $\langle x\rangle$ suggests that this density-matrix element, related to the phases, cannot be properly described by our large- $V$ approach. In the case of an Ohmic bath with exponential cutoff, a transition from a coherent (weakly damped oscillations) regime to an incoherent regime (overdamped decay) can be observed as the system-bath-coupling is increased. ${ }^{8}$ This transition also occurs when the Debye spectral density is used, as shown in Fig. 7. The characteristics of the spectral density are given mainly by its behavior at small frequencies, and both the cutoff Ohmic and Debye spectral densities are proportional to $\omega$ at low frequencies.

A consequence of the structure of the stochastic equations of motion, when $\Delta G^{0}=0$, is that $\lim _{t \rightarrow \infty}\langle z\rangle$ $=\lim _{t \rightarrow \infty}\langle y\rangle=0$, as physically required. The demonstration of this feature is obtained by exchanging $|0\rangle$ and $|1\rangle$ in Eqs. (29)-(32) and setting the time derivatives to zero. This result holds for all values of $V$ and indicates that the prediction of $\langle z(t)\rangle$ 's behavior from the stochastic equations may be reasonable even for the fast-bath regime. On the other hand, the limitations of our procedure to describe the long-time dynamics of $\langle z(t)\rangle$ for a fast bath will be evident for asymmetric reactions, $\Delta G^{0} \neq 0$, as will be discussed below (cf. Fig. 10).

While the stochastic equations of motion do not give correct long-time results for small values of $V$, they can still provide useful information once $E_{r}$ is sufficiently large. In this regime, one anticipates that the initial decay of the reactant state's population will be exponential and the decay rate will be given by the Golden Rule ${ }^{1,2}$

$$
k_{0 \rightarrow 1}=V^{2} \sqrt{\frac{\pi}{E_{r} k_{B} T}} \exp \left(-\frac{\left(E_{r}+\Delta G^{0}\right)^{2}}{4 E_{r} k_{B} T}\right) .
$$

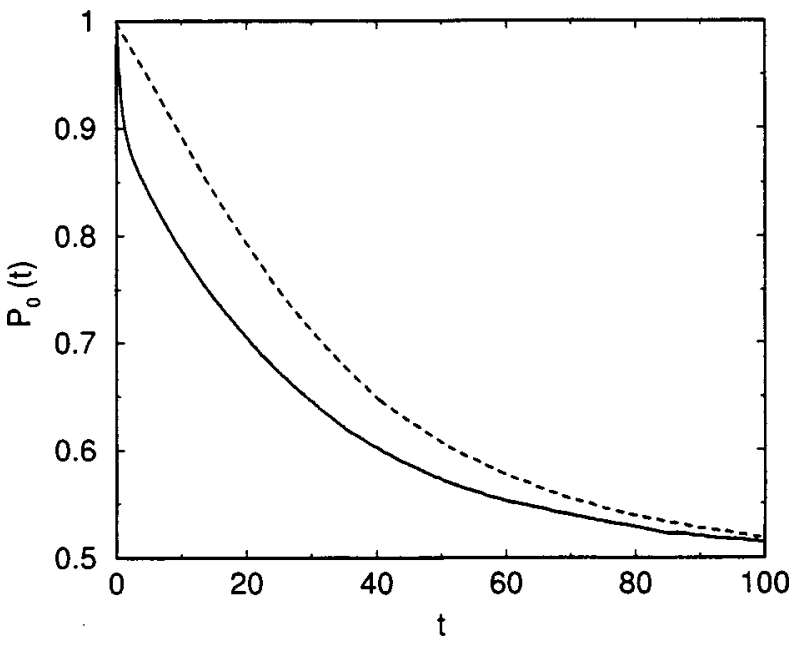

FIG. 9. $P_{0}(t)$ for $\widetilde{V}=1, \widetilde{E}_{r}=40.0, \widetilde{T}=4.0, \Delta \widetilde{G}^{0}=0.0$, and $\alpha=1.0$ (dashed line) and $\alpha=0.0$ (solid line). The short time decay for $\alpha=1.0$ and the short time decay after the transient for $\alpha=0.0$ are well described by the Golden Rule expression. For longer times, the numerical results approach the equilibrium value. The transient behavior (up to $\approx 2$ time units) for $\alpha$ $=0.0$ is described by an activationless Golden Rule expression due to the relatively large initial population at the crossing of the surfaces (see text).

In Fig. 8, we plot the short time reactant state population decays for $V=0.2$ and the solvent equilibrium $(\alpha=1.0)$ and nonequilibrium $(\alpha=0.0)$ initial conditions. The equilibrium one first has a $V$-related fast decay (not visible on the scale of the figure) followed by a decay that agrees with the GR. The nonequilibrium one has a transient behavior for about two time units and then decays with a slope that is quite close to the GR prediction. If $V$ is increased but still kept smaller than the temperature, the Golden Rule should still be an accurate description of the initial population decay. In Fig. 9 we compare the GR with the solutions of the stochastic equations for $V=1.0$ and for $\alpha=1.0$ and $\alpha=0.0$. The same behavior as in Fig. 8 is found. Interestingly, in the nonequilibrium case, the decay of the fast transient approximately goes with the activationless GR rate. With this nonequilibrium initial condition, there are many solvent configurations centered around the crossing of the two diabatic surfaces, so the initial time behavior effectively appears activationless. The GR rate can also be obtained from the initial decay when $\Delta G^{0} \neq 0$, as shown in Fig. 10, where we have plots for an exothermic $\Delta G^{0}=-6.0$ and an endothermic $\Delta G^{0}=6.0$ reaction. Also shown is the time evolution of the reactant state population $P_{0}(t)=0.5[1+\langle z(t)\rangle]$ based on the solution of the kinetic equations for the populations, with their coefficients given by the forward and backward GR rate constants. Based on the kinetic equation

$$
\dot{P}_{0}(t)=-k_{0 \rightarrow 1} P_{0}(t)+k_{1 \rightarrow 0} P_{1}(t)
$$

with $P_{0}(t)+P_{1}(t)=1$, the equilibrium reactant state populations should be $\approx 0.814(\approx 0.182)$ for $\Delta G^{0}=6.0\left(\Delta G^{0}\right.$ $=-6.0$ ). As noted above, we cannot expect the behavior of our stochastic equations to be correct for small values of $V$, and the error in $\langle z(t)\rangle$ increases as $\Delta G^{0}$ increases. Note that the stochastic equations are useful even in this regime as 


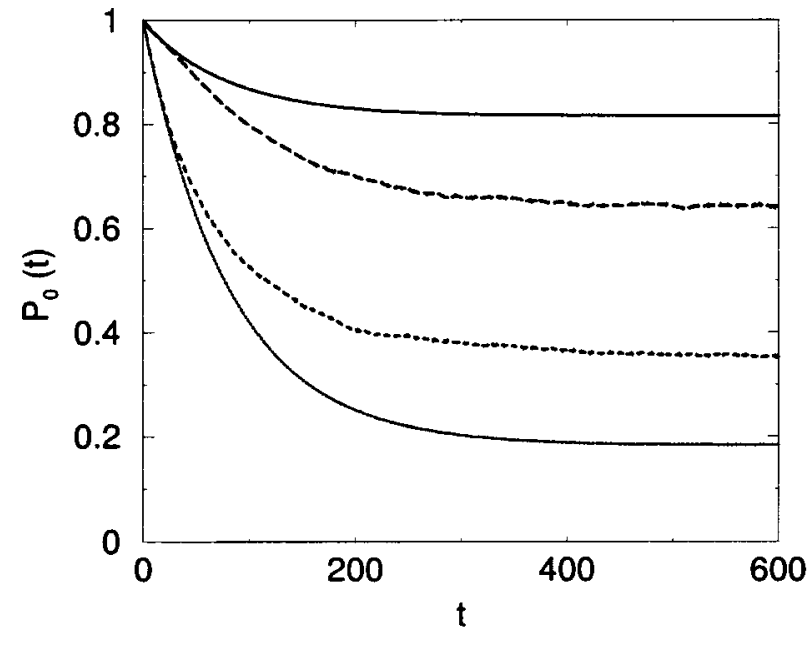

FIG. 10. $P_{0}(t)$ for $\widetilde{V}=0.2, \widetilde{E}_{r}=10.0, \widetilde{T}=4.0, \alpha=1.0, \Delta \widetilde{G}^{0}=6.0$, and $\Delta \widetilde{G}^{0}=-6.0$. The initial decays agree with the Golden Rule expression, but the subsequent time behavior is in error, as seen by comparison with the results based on the kinetic equations (cf. text). Long-dashed $\left(\Delta \widetilde{G}^{0}=6.0\right)$ and dashed $\left(\Delta \widetilde{G}^{0}=-6.0\right)$ lines are for the stochastic equations. Solid $\left(\Delta \widetilde{G}^{0}=6.0\right)$ and dotted $\left(\Delta \widetilde{G}^{0}=-6.0\right)$ lines are for the kinetic equations.

their short time behavior does yield correct forward and backward (using an initial condition of unit population either in the reactant or product state) rate constants, and they can be used in the usual kinetic equations to predict the behavior for all times.

\section{CONCLUDING REMARKS}

The derivation of the stochastic equations of motion presented above was motivated by the important physical regime of photoinitiated electron transfer reactions. These often are characterized by large electronic coupling, strong solute-solvent coupling, low solvent frequencies (for outersphere reactions), and high-temperature, summarized qualitatively by $V \gg \omega_{c}$ and $T \gg \omega_{c}$, and offer the possibility of exploring effects arising from nonequilibrium solvent initial conditions. For large- $V$, guided by the Landau-Zener theory's indication that averaged surfaces are appropriate here, the quantum mechanics of the surface crossing events can be greatly simplified by the effective Hamiltonian in Eq. (6). We previously found this with a one-oscillator analysis ${ }^{21}$ and expect that the approximation would still be valid for a bath of oscillators, though it should be stressed that we know of no proof of this assertion.

The approach we have taken to investigate the long-time consequences of our stochastic equations is to compare them with an expression for the equilibrium density operator that we have obtained by analytic methods under the assumption that the bath variables are classical. [In other work we will show using path integral methods that this equilibrium density operator is the leading term in a perturbation series in $\left(\hbar \omega_{c} / k_{B} T\right)^{2}\left(E_{r} / 2 k_{B} T\right)$.] The resulting density operator yields reactant and product state populations that depend upon $V$. This is a generalization of the small $V$ standard result of chemical kinetics where the equilibrium populations are governed by the exothermicity. It is reassuring that the long-time solutions of the stochastic equations agree with these equilibrium predictions. What is essential for obtaining this behavior is the dynamic feedback of the spin into the bath degrees of freedom, as expressed by the convolution of the bath relaxation $\exp \left(-\omega_{c} t\right)$ and the time dependence of the solute population difference, $z(t)$ [cf. Eq. (12)]. The convolution structure represents the delay in the influence of the constantly changing population difference on the solvent dynamics. The population difference is always being driven by $V$; thus the solvent coordinate must always try to reequilibrate to the instantaneous value of $z(t)$, with the solvent response time measured by $\omega_{c}$.

The competition between the population oscillations induced by $V$ and the damping effects embodied in the $E_{r}$ system-bath-coupling leads to the transition between underdamped and overdamped behavior. If $E_{r}$ is increased sufficiently, $\langle z\rangle$ goes over to essentially an exponential decay regime that can be characterized as a rate process. However, for large- $V$, there is no direct connection of this decay rate to that predicted by the Marcus-Levich expression. ${ }^{2,5}$ A transition between underdamped and overdamped dynamics is well known for small $V^{8-10,15,32}$ and our work shows an analogous transition for large- $V$. The competition between oscillation and decay depends sensitively on the initial preparation of the bath. Our results indicate that for photoinduced electron transfer reactions with $V \gg \omega_{c}$, nonequilibrium initial conditions are more favorable for the observation of oscillations.

The numerical efficiency of our stochastic formulation permits us to readily explore the behavior of the system over a wide range of parameter space. Even though the stochastic equations cannot be correct for all times when $V$ becomes small, they may still provide useful information over a short time regime. Indeed, we showed that the stochastic equations lead to an initial decay of $\langle z(t)\rangle$ that coincides with the predictions obtained from the kinetic equations [cf. Eq. (53)] with coefficients given by the Golden Rule. At longer times, however, the stochastic equation based solutions do not coincide with those of the kinetic equations that must be correct for sufficiently small $V$ and sufficiently large $E_{r}$ (incoherent regime). This becomes especially evident in the equilibrium behavior where the stochastic equations predict populations that contradict the correct values by increasing amounts as $\Delta G^{0}$ increases. The lack of long-time validity of our stochastic equations for small $V$ is not a serious problem since in this regime (and also in the coherent regime of small $\left.E_{r}\right)$ there are several perturbation-theory based methods ${ }^{8-10,15,32}$ that properly describe the time behavior.

In conclusion, the stochastic equations of motion with their feature of the feedback from the system's population difference into the bath mode dynamics provide a reasonable description of the dynamics for all times when $V \gg \omega_{c}$ and $T \gg \omega_{c}$. We are currently exploring the validity of our approximations via a path integral formulation.

\section{ACKNOWLEDGMENTS}

Support by the Center for Fundamental Materials Research at Michigan State University (R.I.C. and M.M.) and the Dirección General de Enseñanza Superior of Spain 
(Project No. PB98-1120) and the Junta de Andalucía is gratefully acknowledged. We also gratefully acknowledge Michael Thorwarts for his help with the implementation of the Makri-Miller procedure.

${ }^{1}$ R. Kubo and Y. Toyozawa, Prog. Theor. Phys. 13, 160 (1955).

${ }^{2}$ V. G. Levich, in Physical Chemistry-An Advanced Treatise, edited by $\mathrm{H}$. Henderson and W. Yost (Academic, New York, 1970), Vol. 9B, p. 985.

${ }^{3}$ J. Ulstrup, Charge Transfer Processes in Condensed Media (Springer, Berlin, 1979).

${ }^{4}$ B. Fain, Theory of Rate Processes in Condensed Media (Springer, Berlin, 1980).

${ }^{5}$ R. A. Marcus and N. Sutin, Biochim. Biophys. Acta 811, 265 (1985).

${ }^{6}$ M. Bixon and J. Jortner, Adv. Chem. Phys. 106, 35 (1999).

${ }^{7}$ A. O. Caldeira and A. J. Leggett, Ann. Phys. (N.Y.) 149, 374 (1983).

${ }^{8}$ A. J. Leggett, S. Chakravarty, A. T. Dorsey, M. P. A. Fisher, A. Garg, and M. Zwerger, Rev. Mod. Phys. 59, 1 (1987).

${ }^{9}$ M. Morillo, R. I. Cukier, and M. Tij, Physica A 179, 411 (1991).

${ }^{10}$ M. Morillo and M. Tij, Physica A 179, 428 (1991).

${ }^{11}$ C. H. Mak and R. Egger, Adv. Chem. Phys. 93, 39 (1996).

${ }^{12}$ W. T. Pollard, A. K. Felts, and R. A. Friesner, Adv. Chem. Phys. 93, 77 (1996).

${ }^{13}$ G. A. Voth, Adv. Chem. Phys. 93, 135 (1996).

${ }^{14}$ N. Makri, Annu. Rev. Phys. Chem. 50, 167 (1999).

${ }^{15}$ M. Grifoni and P. Hänggi, Phys. Rep. 304, 229 (1998).

${ }^{16}$ J. C. Tully, J. Chem. Phys. 93, 1061 (1990).

${ }^{17}$ S. Hammes-Schiffer, J. Phys. Chem. 102, 10443 (1998).

${ }^{18}$ A. A. Golosov, R. Friesner, and P. Pechukas, J. Chem. Phys. 112, 2095 (2000).
${ }^{19}$ M. Morillo and R. I. Cukier, J. Chem. Phys. 110, 7966 (1999).

${ }^{20}$ R. I. Cukier, C. Denk, and M. Morillo, J. Chem. Phys. 111, 5408 (1999).

${ }^{21}$ R. I. Cukier and M. Morillo, Phys. Rev. A 61, 024103 (2000).

${ }^{22}$ N. Sutin, Adv. Chem. Phys. 106, 7 (1999).

${ }^{23}$ K. Wynne and R. M. Hochstrasser, Adv. Chem. Phys. 107, 263 (1999).

${ }^{24}$ A. Lucke, C. H. Mak, R. Egger, J. Ankerhold, J. Stockburger, and H. Grabert, J. Chem. Phys. 107, 8397 (1997).

${ }^{25}$ D. G. Evans, A. Nitzan, and M. A. Ratner, J. Chem. Phys. 108, 6387 (1998).

${ }^{26}$ J. S. Cao, Chem. Phys. Lett. 312, 606 (1999).

${ }^{27}$ Y. S. Jung, R. J. Silbey, and J. S. Cao, J. Phys. Chem. A 103, 9460 (1999).

${ }^{28}$ J. S. Cao, J. Chem. Phys. 112, 4716 (2000).

${ }^{29}$ M. J. Hornbach and Y. Dakhnovskii, J. Chem. Phys. 111, 5073 (1999).

${ }^{30}$ P. J. Reid, C. Silva, P. F. Barbara, L. Karki, and J. T. Hupp, J. Phys. Chem. 99, 2609 (1995).

${ }^{31}$ M. Cho and R. J. Silbey, J. Chem. Phys. 103, 595 (1995).

${ }^{32}$ M. Morillo and R. I. Cukier, Phys. Rev. B 54, 13962 (1996).

${ }^{33}$ N. Makri and D. E. Makarov, J. Chem. Phys. 102, 4600 (1995).

${ }^{34}$ N. Makri and D. E. Makarov, J. Chem. Phys. 102, 4611 (1995).

${ }^{35}$ G. Stock, J. Chem. Phys. 103, 1561 (1995).

${ }^{36}$ U. Müller and G. Stock, J. Chem. Phys. 107, 6230 (1997).

${ }^{37}$ A. A. Golosov, R. Friesner, and P. Pechukas, J. Chem. Phys. 110, 138 (1999).

${ }^{38} \mathrm{H}$. Frölich, Theory of Dielectrics (Oxford University, London, 1958).

${ }^{39}$ N. G. van Kampen, Stochastic Processes in Physics and Chemistry (North-Holland, Amsterdam, 1992).

${ }^{40}$ E. Merzbacher, Quantum Mechanics (Wiley, New York, 1998).

${ }^{41}$ R. L. Honeycutt, Phys. Rev. A 45, 604 (1992).

${ }^{42}$ M. P. Allen and D. J. Tildesely, Computer Simulation of Liquids (Clarendon, Oxford, 1987). 